\title{
Design of an Ultrasensitive Flexible Bend Sensor Using a Silver-Doped Oriented Poly(vinylidene fluoride) Nanofiber Web for Respiratory Monitoring
}

DOI:

10.1021/acsami.9b18823

\section{Document Version}

Accepted author manuscript

Link to publication record in Manchester Research Explorer

Citation for published version (APA):

Jin, L., Zheng, Y., Liu, Z., Li, J., Zhai, H., Chen, Z., \& Li, Y. (2020). Design of an Ultrasensitive Flexible Bend Sensor Using a Silver-Doped Oriented Poly(vinylidene fluoride) Nanofiber Web for Respiratory Monitoring. ACS applied materials \& interfaces, 12(1), 1359-1367. https://doi.org/10.1021/acsami.9b18823

Published in:

ACS applied materials \& interfaces

\section{Citing this paper}

Please note that where the full-text provided on Manchester Research Explorer is the Author Accepted Manuscript or Proof version this may differ from the final Published version. If citing, it is advised that you check and use the publisher's definitive version.

\section{General rights}

Copyright and moral rights for the publications made accessible in the Research Explorer are retained by the authors and/or other copyright owners and it is a condition of accessing publications that users recognise and abide by the legal requirements associated with these rights.

\section{Takedown policy}

If you believe that this document breaches copyright please refer to the University of Manchester's Takedown Procedures [http://man.ac.uk/04Y6Bo] or contact uml.scholarlycommunications@manchester.ac.uk providing relevant details, so we can investigate your claim.

\section{OPEN ACCESS}




\section{Design of ultrasensitive flexible bend sensor using silver-doped oriented PVDF nanofiber web for respiratory monitoring}

$\mathrm{Lu} \mathrm{Jin}^{\dagger}$, Yan Zheng ${ }^{\dagger, \ddagger}$, Zekun $\mathrm{Liu}^{\dagger}$, Jiashen $\mathrm{Li}^{\dagger}$, Heng Zhai ${ }^{\dagger}$, Zhongda Chen ${ }^{\dagger}$ and $\mathrm{Yi} \mathrm{Li}^{\dagger *}$

${ }^{\dagger}$ Department of Materials, School of Natural Sciences, The University of Manchester, Manchester M13 9PL, UK

${ }^{\ddagger}$ School of Fashion, Zhongyuan University of Technology, Zhengzhou 450007, Henan, China

ABSTRACT: We propose a design strategy to fabricate a flexible bend sensor (BS) with ultrasensitivity towards airflow using all PVDF nanofiber web based sensing element and electrode to monitor human respiration. The unique electrospinning (rotational speed of collector of $2000 \mathrm{rpm}$ and tip-to-collector distance of $4 \mathrm{~cm}$ ) with silver nanoparticles interfacing was introduced to prepare an Ag-doped oriented PVDF nanofiber web with high $\beta$-phase content as sensing element (AgOriPVDF, $\beta$-phase crystallinity $44.5 \%$ ). After that, a portion of the prepared AgOriPVDF was processed into flexible and electrically conductive electrode through electroless silver plating technique (SP-AgOriPVDF). Interestingly, the encapsulated AgOriPVDF BS with SPAgOriPVDF electrode exhibited superior piezoelectric bending response (open-circuit peak-to-peak output voltage, $\mathrm{V}_{\mathrm{p}-\mathrm{p}} \approx 4.6 \mathrm{~V}$ ) to injected airflow, which is more than 200 times higher than that of the unpackaged randomly aligned PVDF nanofiber web BS with conductive tape electrode $\left(\mathrm{V}_{\mathrm{p}-\mathrm{p}} \approx 0.02 \mathrm{~V}\right)$. In addition, the factors influencing the 
bend sensitivity of BS such as $\beta$-phase content, nanofiber orientation, flexibility of electrode and so forth were thoroughly analyzed and then discussed. We also demonstrated that the AgOriPVDF BS has sufficient capability to detect and identify various respiratory signal, presenting a great potential for wearable applications, e.g. smart respiratory protective equipment.

KEYWORDS: bend sensor design, poly(vinylidene fluoride), nanofiber, electrospinning, electroless silver plating, respiration

\section{INTRODUCTION}

Self-powered and low-powered sensors are the key components for next-generation wearable devices and the demand of such sensors is increasing rapidly. To meet the growing requirement, various piezoelectric sensors have been extensively studied for sensing application ${ }^{1-3}$. In particular, poly(vinylidene fluoride) (PVDF) has attached special attention as a sensing element in view of its high sensitivity, ultra-low power consumption, excellent flexibility and ease processability ${ }^{4-6}$, showing great merits in the area of wearable applications. PVDF polymer has three stable crystalline phases i.e. $\alpha, \beta$ and $\gamma$ phases. Among them, $\beta$-phase is the most electrically active phase, which presents the highest electrical dipole moment, and thus it is the main source of piezoelectricity ${ }^{7}$. The enhancement of the $\beta$-phase content of PVDF is, therefore, of paramount importance. Piezoelectric PVDF material with high $\beta$-phase content as well as in-situ polarization, i.e. PVDF nanofiber web, can be easily obtained through a straightforward, scalable and cost effective electrospinning technique ${ }^{8-10}$. Nevertheless, 
there are several parameters affecting the $\beta$-phase formation during electrospinning such as additive, tip-to-collector distance (TCD), rotational speed of collector (RSC), applied voltage, injection flow rate and so forth ${ }^{11-13}$. Especially, doping additive such as carbon nanotube ${ }^{14}$, nanocaly ${ }^{15}$ and graphene ${ }^{16}$ into PVDF electrospinning solution is one of the effective ways to further increase the $\beta$-phase content since these nanomaterials act as nucleation agents to promote the $\beta$-phase formation.

However, the higher $\beta$-phase content cannot ensure the better bend sensitivity of PVDF nanofiber web ${ }^{1,5}$, because some other factors could affect the sensitivity of bend sensor (BS) as well. To be specific, owing to randomly oriented nanofiber web structure, the bending force cannot be sufficiently applied to each nanofiber to make them deformed, causing the lower change in dipole moment ${ }^{5}$. Therefore, it is expected that the oriented PVDF nanofiber web presents better bend sensitivity than the random oriented one. There are several ways such as magnetic electrospinning ${ }^{17}$ and high RSC ${ }^{18}$ methods to obtain aligned nanofibers via electrospinning. The former requires strong magnetic field by two paralleled permanent magnets to order the nanofibers when electrospinning, but this could disorder the oriented dipoles of PVDF nanofiber web. Therefore, high RSC method is a more practical way to fabricate oriented PVDF nanofiber web.

More importantly, unlike the pressure sensor, the BS generates electric signal by bending itself. The flexibility of BS, especially the elasticity of electrode, plays a crucial role in the bend sensitivity. There are also the so-called flexible electrodes reported such as conductive tapes ${ }^{5,19-21}$ and conductive fabrics ${ }^{15,22,23}$ for the PVDF nanofiber web 
based pressure sensors. However, the flexibility of such conductive tapes and fabrics is still much higher than that of PVDF nanofiber web. Furthermore, due to the porous structure of nanofiber web, it is rather difficult to coat conductive pastes or inks on both sides of PVDF nanofiber web as electrodes. Therefore, fabricating an appropriate electrode for the PVDF nanofiber web sensing element is one of challenges in the design of high performance BS.

Overall, the design strategy of BS with PVDF nanofiber web is definitely different with that of the pressure sensor. The factors such as nanofiber orientation and flexibility of electrode could be minor important for the pressure sensor, while they become decisive factors when designing and developing the BS. Unfortunately, most previous research regarding PVDF nanofiber web emphasized on pressure sensors or generators rather than BSs.

Hence, this study focused on designing ultrasensitive BS with PVDF nanofiber web and illustrated the underlying mechanism of the increased bend sensitivity of the BS via analyzing factors i.e. $\beta$-phase content, nanofiber orientation and flexibility of electrode. Taking into account of these factors, we introduced a novel approach to design an BS using all silver nanoparticles doped oriented PVDF nanofiber web based sensing element and electrode for respiratory monitoring. Consequently, the newly developed BS exhibited superior bend sensitivity towards airflow in comparison to the conventional BS with the randomly aligned PVDF nanofiber web and conductive tape electrode, importantly the piezoelectric bending response was improved more than 200 times. We also demonstrated that the developed BS has sufficient capability to record 
and identify the various breathing patterns. These findings can be used to develop a cost effective, low-powered and high performance wearable respiration sensor for smart respiratory protective equipment.

\section{EXPERIMENTAL SECTION}

Materials. PVDF pellets $\left(\mathrm{M}_{\mathrm{w}} \approx 275\right.$ 000, Sigma-Aldrich, UK), sodium hydroxide (Sigma-Aldrich, UK), dextrose (Sigma-Aldrich, UK) and ammonia solution (SigmaAldrich, UK), silver nitrate (Fisher Scientific, UK), N,N-dimethylformamide (DMF, Fisher Scientific, UK), acetone (Fisher Scientific, UK), conductive tape (Nickle/copper coated woven polyester fabric with acrylic adhesive, Amazon, UK) and Ecoflex ${ }^{\mathrm{Tm}} 00$ 30 (Smooth-On Inc, USA).

Electrospinning for PVDF Nanofiber Web Preparation. PVDF pellets were dissolved in a binary solvent system of DMF/Acetone (4/6, v/v) to prepare PVDF electrospinning solution (10 wt. \%). Then, 1 wt. \% of silver nitrate compared to the PVDF weight was added to the PVDF solution by stirring for $4 \mathrm{~h}$ to form silver nanoparticles reduced by DMF. Another solution was also prepared, in which silver nanoparticles were not added, for reference experiment. After that, the prepared solutions were filled into a $10 \mathrm{~mL}$ syringe with a needle of 23 gage. The electrospinning was performed at an injection rate of $0.5 \mathrm{~mL} / \mathrm{h}$ and an applied voltage of $16 \mathrm{kV}$. Silverdoped oriented PVDF nanofiber web (AgOriPVDF) was fabricated at RSC of $2000 \mathrm{rpm}$ and TCD of $4 \mathrm{~cm}$ for the sensing element as well as the electrode and randomly aligned neat PVDF nanofiber web (NeatPVDF) was made at a low RSC of $5 \mathrm{rpm}$ at a larger TCD of $10 \mathrm{~cm}$ for reference sensing element. Electrospinning of each sample was 
conducted for $8 \mathrm{~h}$ to obtain uniform thickness of $\sim 100 \mu \mathrm{m}$ of PVDF nanofiber web.

Electroless Silver Plating Process for Electrode Preparation. Electroless silver plating process requires four solutions i.e. a silver nitrate solution $(0.1 \mathrm{M})$, a sodium hydroxide solution (0.8 M), a dextrose solution (0.12 M) and an ammonia solution (15 M). First, $40 \mathrm{~mL}$ of silver nitrate solution was mixed with $20 \mathrm{~mL}$ of sodium hydroxide solution with stirring. Once brown precipitate formed, the ammonia solution was added until it was dissolved to produce a water-soluble diamine silver complex compound. After that, the diamine silver complex solution was poured into a beaker with AgOriPVDF and the dextrose solution $(4 \mathrm{ml})$ was added into the beaker. The diamine silver complex cation ([Ag( $\left.\left.\left(\mathrm{NH}_{3}\right)_{2}\right]^{+}\right)$was reduced gradually by dextrose to silver, which was deposited preferentially on AgOriPVDF surface instead of the wall of beaker. The reduction was conducted for 30 minutes. It is noteworthy to mention that the PVDF nanofiber web should be immersed into ethanol for $24 \mathrm{~h}$ before silver plating to enhance their wettability towards the aqueous electroless silver plating solution. This provides uniform silver plating from the surface to deep inside of the PVDF nanofiber web.

Characterizations. The formation of crystalline phases in PVDF nanofiber web was determined by Fourier transform infrared (FTIR, Perkin Elmer Spectrum 1000) spectroscopy in the range between 400 and $1500 \mathrm{~cm}^{-1}$ with a resolution of $4 \mathrm{~cm}^{-1}$. The crystalline structure of PVDF nanofiber web was analyzed by X-ray diffraction (XRD, Bruker, D8 Advance) in the $2 \theta$ range of $10^{\circ}$ to $40^{\circ}$ with a $\mathrm{CuKa}(\lambda=1.54 \AA)$ radiation source under an operating $40 \mathrm{kV}$ voltage and $40 \mathrm{~mA}$ current. The surface morphology of PVDF nanofiber web was analyzed with a field emission scanning electron 
microscope (FE-SEM) at an acceleration voltage of $1.5 \mathrm{kV}$. Furthermore, the statistical distributions of alignment angle and the fiber diameter were calculated using ImageJ software. The stress-strain and durability measurement were performed through an electromechanical universal testing system (Instron, 3400) to examine the elastic modulus of BS. The sheet resistance of the electrode was measured by a Keithley 2000 digital multimeter.

Piezoelectric Bending Response Test. In order to systematically evaluate the piezoelectric bending response towards airflow, a respiration simulator was assembled as illustrated in Figure 1. The main part of this simulator is an air pump, which simulates the human lung function. The air pump comprises two taps, i.e. an inlet tap and an outlet tap, and the outlet tap is connected to a sensor holder with an orifice in center. The orifice dimension (width/length) is $15 \mathrm{~mm} / 25 \mathrm{~mm}$ so that the bend sensor (20 mm / $40 \mathrm{~mm}$ ) could cover the orifice and thereby the injected air can deflect the sensor. In addition, the open-circuit output voltage corresponding to injected airflow was measured using a Biopac system (MP160 and HLT1000, Biopac, USA) and a Piezo film lab amplifier under the conditions (voltage mode, input impedance $=100 \mathrm{M} \Omega$, gain $=20 \mathrm{~dB}$, band-pass filter $=0.1-10 \mathrm{~Hz}$, Measurement Specialties, UAS). 


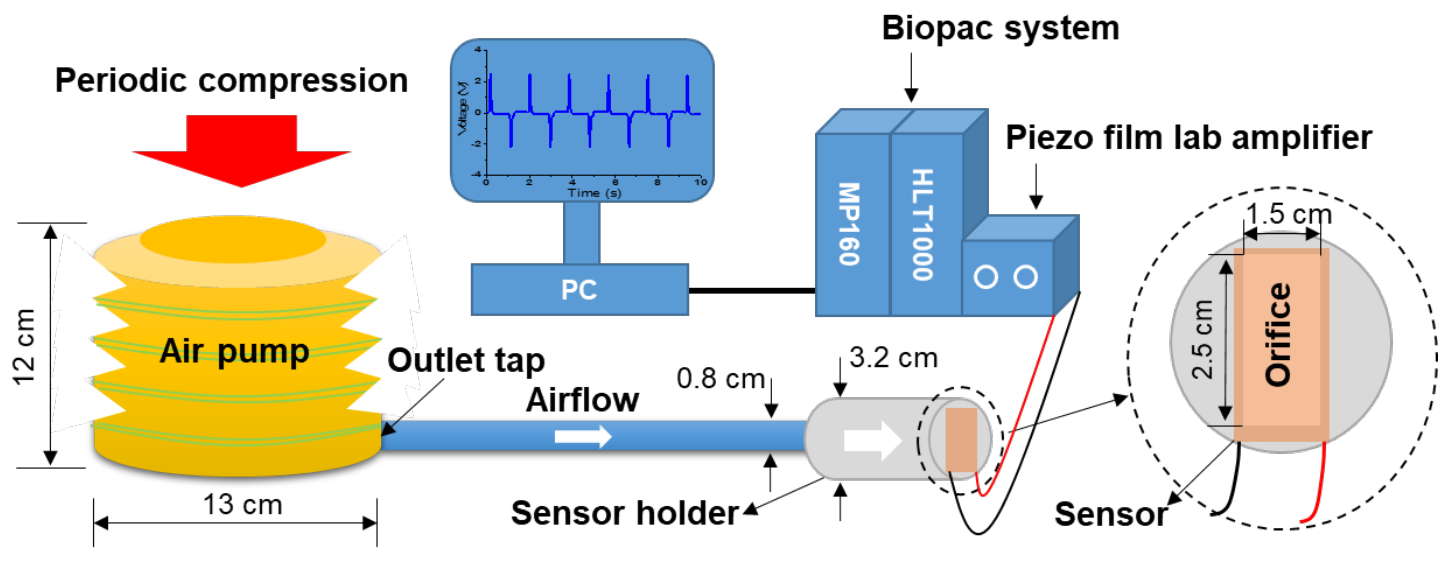

Figure 1. Schematic of the respiration simulator for piezoelectric bending response measurement with a Biopac system and a Piezo film lab amplifier.

\section{RESULTS AND DISCUSSION}

Design Strategy of Bend Sensor. Figure 2a-g illustrate the fabrication strategy procedure used to make an ultrasensitive and flexible BS. In order to address the abovementioned issues in designing of BS with PVDF nanofiber web, we manufactured the BS according to the following steps. It starts with AgOriPVDF preparation via the unique electrospinning ( $\mathrm{RSC}=2000 \mathrm{rpm}$ and $\mathrm{TCD}=4 \mathrm{~cm}$, Figure 2a) with silver nanoparticle interfacing. Then the AgOriPVDF is cut into two parts (Figure 2b), one is utilized as a sensing element and the other one is for electrode after electroless silver plating (Figure 2c). After that, silver plated AgOriPVDFs (SP-AgOriPVDF, Figure 2d) are laminated on both sides of AgOriPVDF as electrodes (Figure 2e). Finally, the whole assembled structure is encapsulated with Ecoflex as a protecting layer (Figure 2f). Finally, Figure 2g shows a digital photograph of AgOriPVDF SB with SP-AgOriPVDF electrode. 


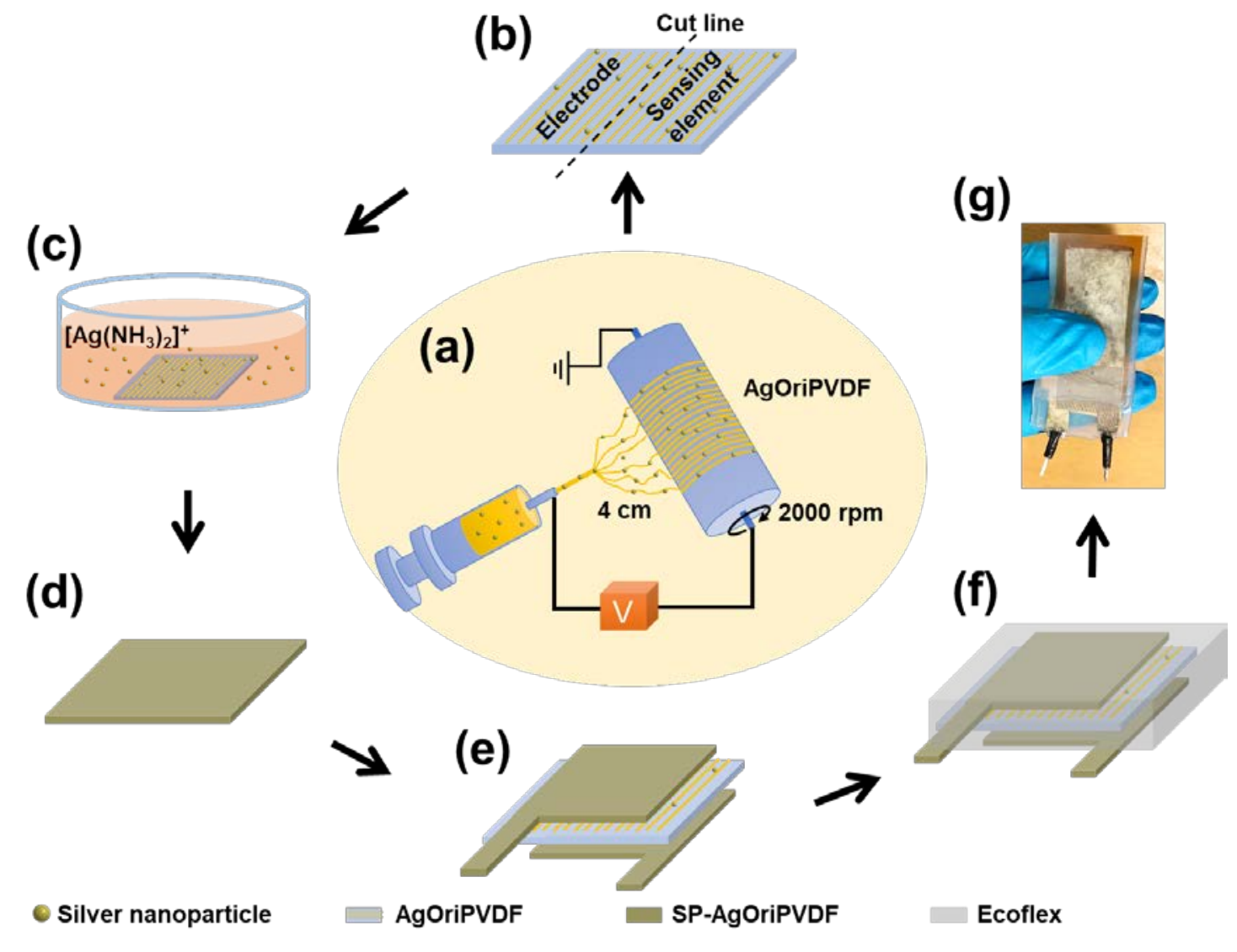

Figure 2. Schematic of ultrasensitive and flexible BS design using PVDF nanofiber web. (a) Unique electrospinning (RSC $=2000 \mathrm{rpm}$ and TCD $=4 \mathrm{~cm}$ ) with silver interfacing for AgOriPVDF preparation, (b) two different purposes of AgOriPVDF with cut line, (c) electroless silver plating process, (d) SP-AgOriPVDF, (e) lamination process, (f) Ecoflex encapsulation, (g) photograph of AgOriPVDF SB with SPAgOriPVDF electrode.

Crystalline Phase Identification. The crystalline phases of the prepared PVDF nanofiber webs were identified by XRD patterns and FTIR spectra, and the corresponding crystallinity was evaluated from the curve deconvoluated XRD patterns. Figure 3a,b display the XRD patterns of NeatPVDF and AgOriPVDF, respectively. The NeatPVDF presents two clear diffraction peaks at $18.3^{\circ}$ ( $\alpha$-phase) and $20.6^{\circ}$ ( $\beta$-phase) whereas the AgOriPVDF only exhibits one sharp diffraction peak at $20.6^{\circ}$ ( $\beta$-phase) ${ }^{24}$. 
This indicates that the NeatPVDF is composed of $\alpha$-phase and $\beta$-phase while the AgOriPVDF mainly consists of $\beta$-phase. This result is further confirmed by the FTIR analysis. In Figure 2c, the NeatPVDF shows two clear $\alpha$-characteristic vibrational bands at $489 \mathrm{~cm}^{-1}$ and $764 \mathrm{~cm}^{-1}$ while they do not appeared in the AgOriPVDF. Moreover, the $\beta$-phase existence of both samples can be evident from the distinct two peaks at $1276 \mathrm{~cm}^{-1}$, whereas the $\gamma$-phase absence is identified from the peaks broadening at $1234 \mathrm{~cm}^{-15}$. The degree of total crystallinity $\left(\mathrm{C}_{\mathrm{t}}\right), \beta$-phase crystallinity $\left(\mathrm{C}_{\beta}\right)$ and $\alpha$-phase crystallinity $\left(\mathrm{C}_{\alpha}\right)$ calculated from the curve deconvoluated XRD patterns (Supporting Information, S1) and displayed in Figure 3a,b. It can be found that not only the $C_{t}$ but also the $C_{\beta}$ of the AgOriPVDF (i.e. $C_{t}$ and $C_{\beta} \sim 44.5 \%$ ) are remarkably higher than those of the NeatPVDF (i.e. $\mathrm{C}_{t} \sim 37.8 \%$ and $\mathrm{C}_{\beta} \sim 33.8 \%$ ), the $\mathrm{C}_{\beta}$ difference can reach $10 \%$.

The enhanced crystallinity is ascribed to two electrospinning parameters i.e. silver nanoparticles and TCD. Incorporation of silver nanoparticles in electrospinning solution notably increased the $C_{t}$ and $C_{\beta}$ of the PVDF nanofiber. This is because the silver nanoparticles can act as nucleation agents to promote the $\beta$-phase formation due to the specific interaction of the surface charge of silver nanoparticles with the $\mathrm{CH}_{2}$ dipoles of the $\beta$-phase ${ }^{25}$. To be more specific, the silver atoms have a large amount of free electrons from their outmost electronic shell, showing electro-negative. As a result, the silver nanoparticles surface can attract the electro-positive $\mathrm{CH}_{2}$ dipoles of the PVDF chain to assist the $\beta$-phase formation as represented in Figure 3d. In addition, TCD used in AgOriPVDF fabrication is around $4 \mathrm{~cm}$, which is the shortest distance for the PVDF 
nanofiber web formation. It significantly increases the electric field, which is 1.5 times higher than that of NeatPVDF $(10 \mathrm{~cm})$. Consequently, the increased electric field more sufficiently promote the dipolar polarization of the PVDF molecular chain to improve the $\beta$-phase content ${ }^{15,24}$. Moreover, the PVDF nanofiber was more efficiently drawn by the electric field force induced during electrospinning to facilitate the transition from $\alpha$-phase into $\beta$-phase formation ${ }^{26}$ as shown in Figure 3e.

Overall, the silver nanparticles doped PVDF nanofiber web with high $\beta$-phase content (i.e. AgOriPVDF, $\mathrm{C}_{\beta} \sim 44.5 \%$ ) has been obtained via electrospinning. The underlying mechanism of the increased $\beta$-phase content has been fully discussed as well. It is expected the AgOriPVDF with higher content should be the better piezoelectricity than NeatPVDF, since it is directly related to the $\beta$-phase content ${ }^{11}$.
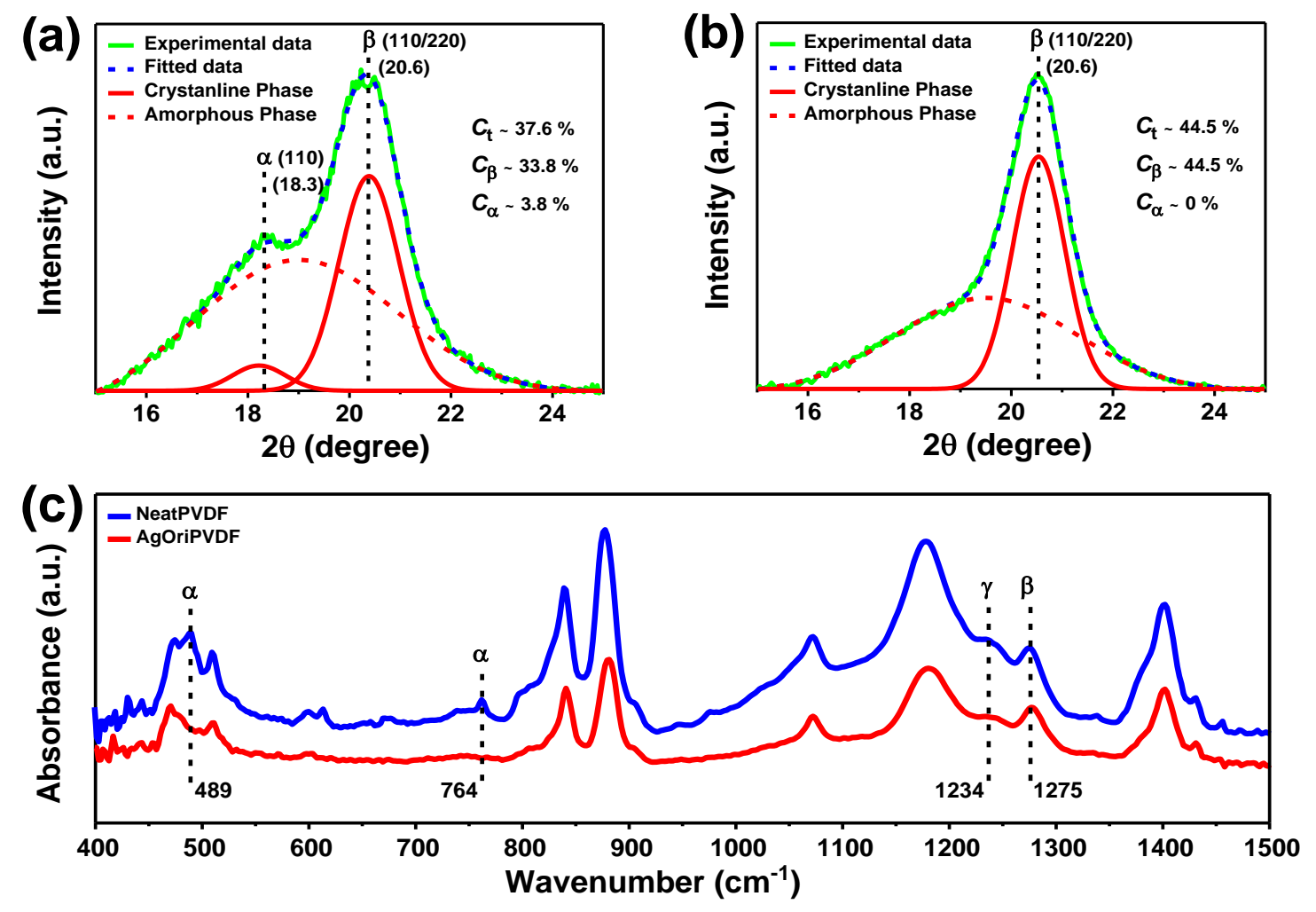
(d)

Silver
nanoparticles
Interaction
$\beta$-phase

While: Hydrogen (e)

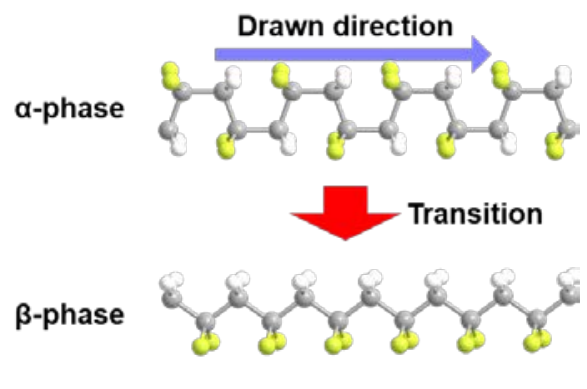

Figure 3. XRD patterns and the corresponding curve deconvolutions of (a) NeatPVDF and (b) AgOriPVDF ranging from $15^{\circ}$ to $25^{\circ}$, (c) FTIR spectra of NeatPVDF and AgOriPVDF in the wavenumber region from $400 \mathrm{~cm}^{-1}$ to $1500 \mathrm{~cm}^{-1}$, and schematic representations of (d) the interaction of the surface charge of silver nanoparticles with the $\mathrm{CH}_{2}$ dipoles of $\beta$-phase and (e) the transition from $\alpha$-phase to $\beta$-phase.

Morphological Analysis. The surface morphology of the prepared PVDF nanofiber webs was investigated via digital photographs and FE-SEM images. Figure 4a,b display photographs of NeatPVDF and AgOriPVDF, respectively. Compared with the white NeatPVDF, the AgOriPVDF shows golden color. Initially, the AgOriPVDF showed light yellow just after electrospinning and it gradually became deep golden color over time, because some silver nanoparticles deposited on the nanofiber surface were oxidized. The AgOriPVDF is not electrically conductive, as the silver nanoparticles have not connected with each other due to the limited amount. These silver nanoparticles on the nanofiber surface, however, play an important role in the electroless silver plating process since they can serve as catalysts to attract the reduced silver atoms during silver plating. The inset of Figure $4 \mathrm{~b}$ shows a photograph of SPAgOriPVDF, presenting silver color due to silver nanoparticles on the nanofiber web 
surface. Figure 4c,d display the respective FE-SEM images of NeatPVDF and AgOriPVDF. The bead defect free PVDF nanofibers were observed in both FE-SEM images. The nanofibers of NeatPVDF show randomly aligned with an average diameter of $70 \mathrm{~nm}$ (Figure 4a, left down inset) whereas most nanofibers in AgOriPVDF are oriented with an average diameter of $50 \mathrm{~nm}$ (Figure 4b, left down inset). Besides, in order to quantitatively evaluate the nanofiber orientations of two samples, the alignment angle distributions were calculated using ImageJ program. There is no obvious peak in the nanofiber alignment angle distribution of NeatPVDF (Figure 4a, right upper inset) while that of AgOriPVDF exhibits a sharp peak at around $90^{\circ}$ (Figure 4b, right upper inset), indicating AgOriPVDF was uniaxially oriented when collected. This is mainly responsible for the high RSC (2000 rpm). Figure 4e,f present FE-SEM images of SPAgOriPVDF with different magnifications. Silver nanoparticles are uniformly deposited on the surface of nanofiber web and firmly connected with each other, guaranteeing the excellent electrical conductivity of SP-AgOriPVDF. The durability of SP-AgOriPVDF was also evaluated through the cyclic fatigue bending testing (3000 cycles). According to a FE-SEM image (Supporting Information, Figure S1), there is no significant change in the surface of SP-AgOriPVDF after 3000 cycles bending.

Overall, a highly oriented Ag-doped PVDF nanofiber web, i.e. AgOriPVDF, was prepared at high RSC of $2000 \mathrm{rpm}$. It is expected that the aligned AgOriPVDF should exhibit better bend sensitivity than NeatPVDF because the nanofiber orientation is one of decisive factors for the bend sensitivity 

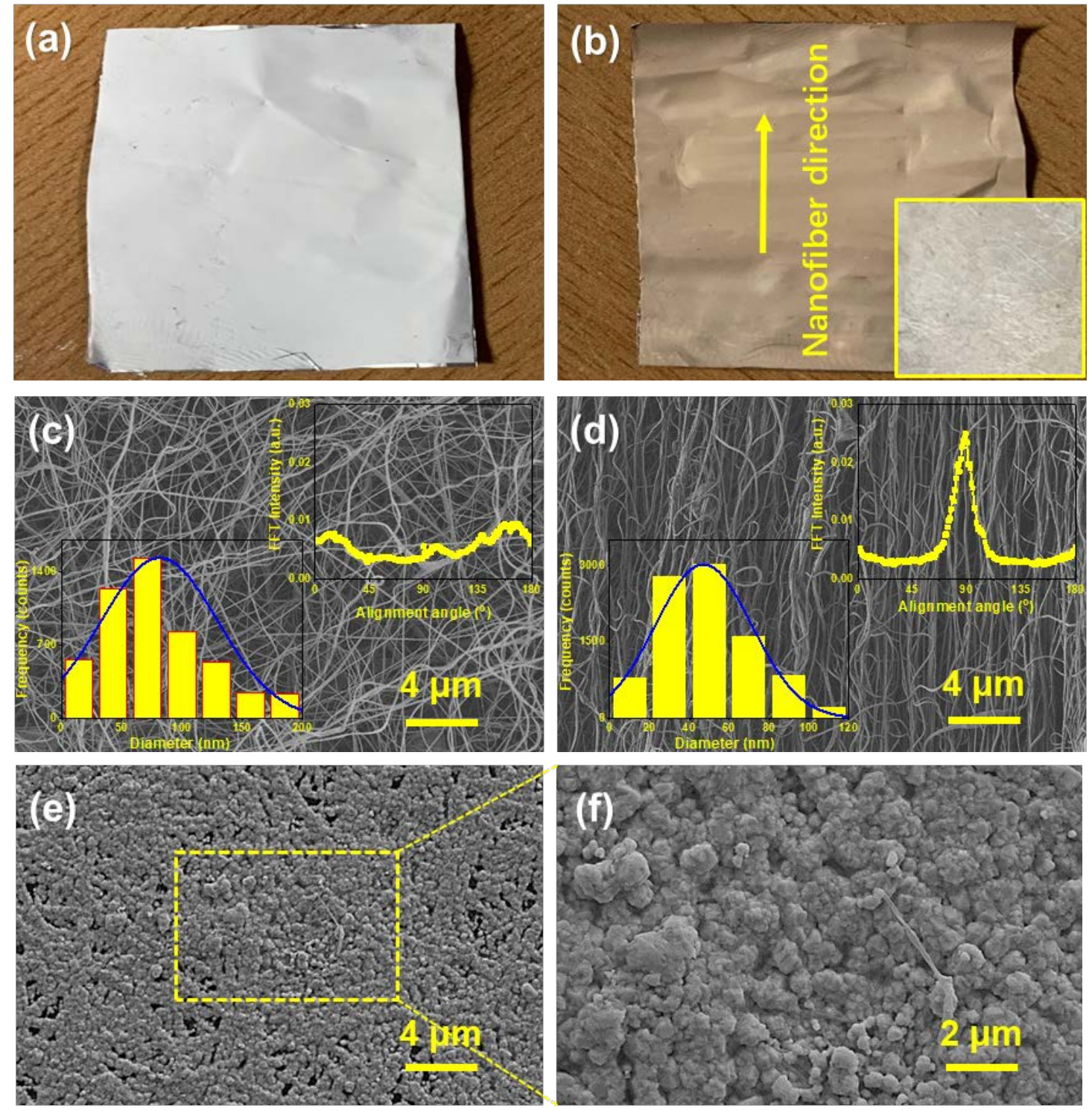

Figure 4. Photographs of (a) NeatPVDF and (b) AgOriPVDF with an inset of SPAgOriPVDF. FE-SEM images (5000 magnifications) with the statistical distributions of nanofiber diameter (left down insets) and nanofiber alignment angle (right upper insets) of (c) NeatPVDF and (d) AgOriPVDF. FE-SEM images of SP-AgOriPVDF with (e) 5000 magnifications and (f) 20000 magnifications.

Bend Sensitivity Evaluation. To evaluate and compare the bend sensitivity towards airflow, a combination of the respiration simulator and Instron was employed (Supporting Information, Figure S2). When periodic pressure is applied to the air pump 
by Instron, the simulator injected an equivalent amount of air to bend the sensor each time, allowing the sensor to be tested at the same condition. To be specific, the opencircuit output voltage measured is related not only to the injected air volume but also to the injection time, i.e. airflow rate. Since both injected air volume (250 mL) and injection time $(0.5 \mathrm{~Hz})$ are constant each time, the airflow rate profile from the respiration simulator is identical when ignoring air compression. Consequently, the open-circuit output voltage corresponding to injected airflow rate indirectly reflects the bend sensitivity of the BS. To simplify the bend sensitivity evaluation, the open-circuit peak-to-peak output voltage $\left(\mathrm{V}_{\mathrm{p}-\mathrm{p}}\right)$ is measured and compared in this study ${ }^{27}$.

First, the effect of sensing elements i.e. NeatPVDF and AgOriPVDF on the bend sensitivity was assessed by simply attaching conductive tapes on both sides of two PVDF nanofiber webs as electrodes without packaging. Figure 5a,b compare the $\mathrm{V}_{\mathrm{p}-\mathrm{p}}$ of the NeatPVDF and the AgOriPVDF. As expected, the AgOriPVDF exhibits a $V_{\mathrm{p}-\mathrm{p}}$ of $0.18 \mathrm{~V}$ whereas the NeatPVDF presents a significantly low $\mathrm{V}_{\mathrm{p}-\mathrm{p}}$ of $0.02 \mathrm{~V}$, which means the bend sensitivity of the AgOriPVDF could be nine times of that of NeatPVDF. Then, two Ecoflex encapsulated AgOriPVDF BSs were fabricated with different electrodes, i.e. SP-AgOriPVDF electrode and conductive tape electrode, to evaluate the impact of various electrodes on the bend sensitivity. As shown in Figure 5c,d, the AgOriPVDF BS with SP-AgOriPVDF electrode $\left(\mathrm{V}_{\mathrm{p}-\mathrm{p}} \approx 4.6 \mathrm{~V}\right)$ exhibits more than double higher piezoelectric bending response than the one with conductive tape electrode $\left(\mathrm{V}_{\mathrm{p}-\mathrm{p}} \approx 1.4\right.$ V). Moreover, compared to the unpackaged AgOriPVDF BS $\left(\mathrm{V}_{\mathrm{p}-\mathrm{p}} \approx 0.18 \mathrm{~V}\right)$, the piezoelectric response of the Ecoflex encapsulated AgOriPVDF BS with conductive 
tape electrode $\left(\mathrm{V}_{\mathrm{p}-\mathrm{p}} \approx 1.4 \mathrm{~V}\right)$ remarkably increase, which can reach seven times. It is also apparent that packaging the BS with Ecoflex results in the better bend sensitivity. In order to evaluate the long-term stability and durability of the AgOriPVDF BS with SP-AgOriPVDF electrode, cyclic fatigue testing was performed over a prolonged period of time (6000 s) under the same condition (Figure 5e). The stable and continues piezoelectric bending response without any significant variation toward injected airflow demonstrates its great durability and stability.

As long as a pressure is applied to the air pump, the respiration simulator injects a relevant amount of air to the bend sensor. This produces differential pressure between front side and back side of the BS to deflect the sensor, thereby generating electrical signal. To be specific, the electroactive $-\mathrm{CH}_{2}-/-\mathrm{CF}_{2}$ - dipoles in $\beta$-phase are polarized and oriented along the thickness direction by the applied voltage during electrospinning (Figure 6a, dash line) ${ }^{21}$. When a bending force induced by differential pressure is applied to the sensor, the front side of the sensor facing airflow receives a tensile force. This increases the length of the front side, resulting in the decreased density of positive charge. On the contrary, the opposite side is subjected to compressive force, leading to the elevated density of negative charge. This density change in electrical charge of two sides causes a current during bending (Figure 6a, solid line). Obviously, the higher $\beta$ phase content, i.e. $-\mathrm{CH}_{2}-/-\mathrm{CF}_{2}$ - dipoles content, of PVDF nanofiber web produces much larger change in the electrical charge when bending, presenting the higher piezoelectric sensitivity. Besides, as the aligned nanofibers can be subjected to more intensive tensile and compressive force when bending in comparison to the randomly oriented 
nanofibers as illustrated in Figure 5b, the former can present the better bend sensitivity. It is the underlying mechanism why the high $\beta$-phase content and aligned AgOriPVDF exhibits significantly higher bend sensitivity than the randomly oriented NeatPVDF. To further investigate the contribution of two factors to the increased bend sensitivity, a silver doped but randomly oriented PVDF nanofiber web (AgRanPVDF, $\mathrm{C}_{\beta} \sim 43.9 \%$ ), which has similar $C_{\beta}$ to that of AgOriPVDF, was fabricated again for extra bend sensitivity experiment (Supporting Information, Figure S3). The piezoelectric bending response of AgRanPVDF $\left(\mathrm{V}_{\mathrm{p}-\mathrm{p}} \approx 0.04 \mathrm{~V}\right)$ is two times of NeatPVDF $\left(\mathrm{V}_{\mathrm{p}-\mathrm{p}} \approx 0.02 \mathrm{~V}\right)$ while the piezoelectric response of $\operatorname{AgOriPVDF}\left(\mathrm{V}_{\mathrm{p}-\mathrm{p}} \approx 0.18 \mathrm{~V}\right)$ is more than four times of that of AgRanPVDF, which means the increased bend sensitivity by nanofiber orientation is over double of that by the $\beta$-phase content. Therefore, for a PVDF nanofiber based SB, the orientation of PVDF nanofiber is a decisive factor for its bend sensitivity, it is probably more important than the $\beta$-phase content. Similarly, after Ecoflex encapsulation, the bending force can more efficiently propagate into each nanofiber to be deformed. Therefore, packaging BS with elastic materials, e.g. Ecoflex, is quite effective way to improve the bend sensitivity and enhance the durability of BS together.

There are two factors of the electrode i.e. flexibility and electrical conductivity, which probably influence the bend sensitivity. Since the BS is bent its body to generate signal in response to external force, i.e. airflow, the total elasticity of BS is a decisive factor. Figure 6c shows the elastic modulus, E, of two AgOriPVDF BSs with different electrodes. It is noteworthy that the stress-strain curve of BS with conductive tape 
electrode exhibits clear hysteresis, showing the different Es between compressing and releasing processes. The $\mathrm{E}$ of $\mathrm{BS}$ with conductive tape electrode (E $\sim 0.42 \mathrm{KPa}$, which is the releasing stage) is significantly higher than that with SP-AgOriPVDF electrode (E $\sim 0.08 \mathrm{KPa})$. Such flexibility difference between two materials is responsible for the increased bend sensitivity. In addition, as the lower electrical conductivity of electrode can cause the electrical charge loss, the conductivity of electrode is important. Figure 6d shows the sheet resistances of two electrode materials i.e. SP-AgOriPVDF and conductive tape. The sheet resistance of SP-AgOriPVDF shows about $27.7 \pm 4.9 \Omega / \mathrm{m}^{2}$, which is in the range of $22-51 \Omega / \mathrm{m}^{2}$ that Logeeswaran et al. ${ }^{28}$ reported. It is more than four times of that of conductive tape $\left(125 \pm 5.0 \Omega / \mathrm{m}^{2}\right)$, probably resulting in the improved bend sensitivity of AgOriPVDF BS with SP-AgOriPVDF electrode. To further determine the contribution of two factors of the electrode i.e. flexibility and conductivity to the elevated bend sensitivity, an additional experiment was carried out as well. Since the impact of the flexibility of electrode on the pressure sensitivity is limited, the conductivity is the only factor affecting the pressure sensitivity of two BSs. As presented in Figure S4, the piezoelectric pressuring response of AgOriPVDF BS with SP-AgOriPVDF electrode $\left(\mathrm{V}_{\mathrm{p}-\mathrm{p}} \approx 0.20 \mathrm{~V}\right.$,) is slightly higher than that with conductive tape electrode $\left(\mathrm{V}_{\mathrm{p}-\mathrm{p}} \approx 0.17 \mathrm{~V}\right)$. This means the difference in electrical charge loss due to the electrode conductivity of two BSs is not significant, implying the effect of electrode conductivity on the bend sensitivity is finite; the flexibility is the main contributor.

Overall, a high performance encapsulated AgOriPVDF BS with SP-AgOriPVDF 
electrode was successfully fabricated and demonstrated. As shown in Figure 5. its piezoelectric bending response $\left(\mathrm{V}_{\mathrm{p}-\mathrm{p}} \approx 4.6 \mathrm{~V}\right)$ is over 3 times of that with conductive fabric electrode ( $\left.\mathrm{V}_{\mathrm{p}-\mathrm{p}} \approx 1.4 \mathrm{~V}\right)$, is about 25 times of unpackaged AgOriPVDF BS with conductive tape electrode $\left(\mathrm{V}_{\mathrm{p}-\mathrm{p}} \approx 0.18 \mathrm{~V}\right)$ and is 230 times of the randomly orientated and unpackaged NeatPVDF BS with conductive tape electrode $\left(\mathrm{V}_{\mathrm{p}-\mathrm{p}} \approx 0.02 \mathrm{~V}\right)$. Moreover, the factors affecting the bend sensitivity of BS was fully investigated. For sensing element, nanofiber orientation and $\beta$-phase content of PVDF nanofiber web play crucial roles in the bend sensitivity. The flexibility of electrode is key parameter for the bend sensitivity as well.
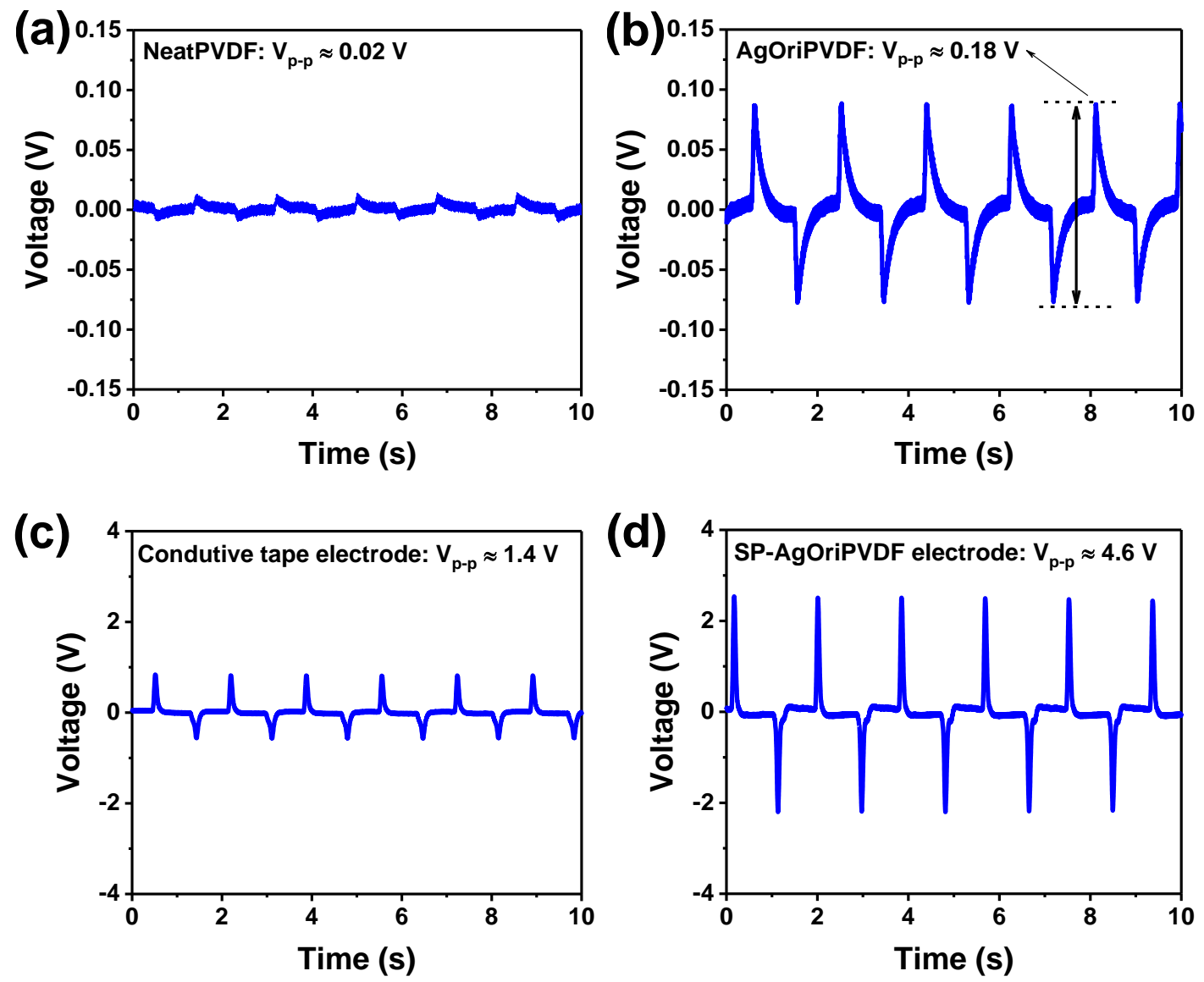


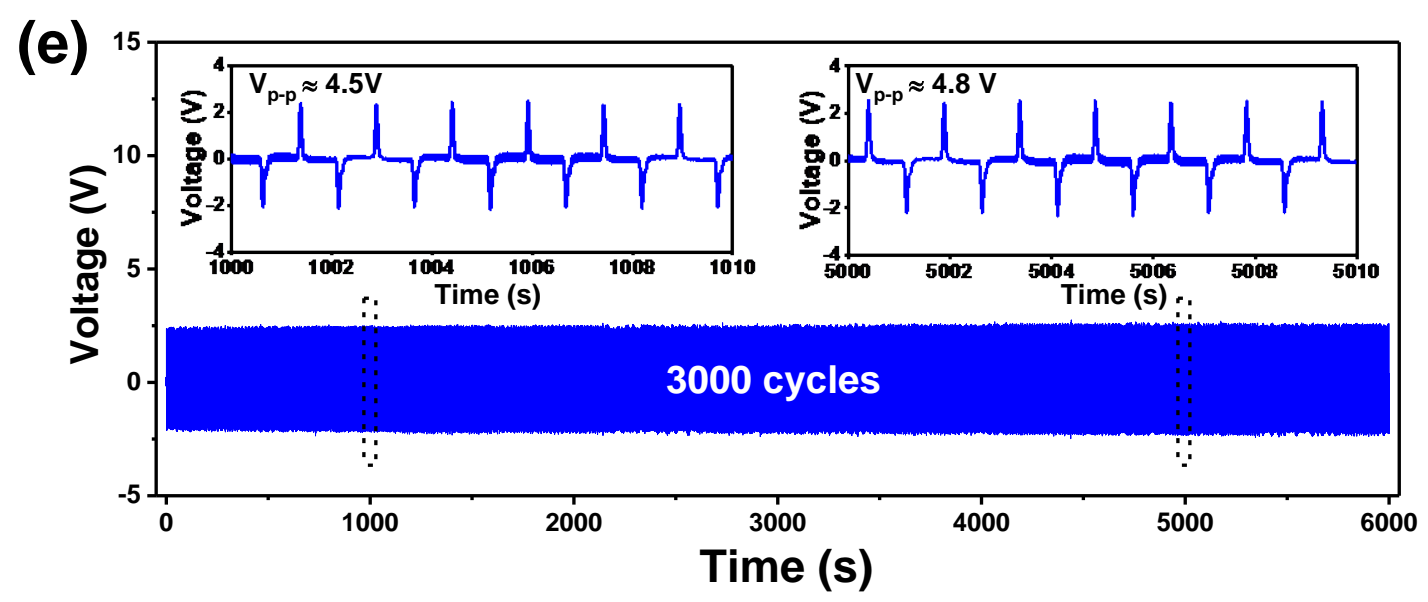

Figure 5. Comparisons of piezoelectric bending responses of (a) NeatPVDF and (b) AgOriPVDF sensing elements both with conductive tapes as electrodes without packaging, and two Ecoflex encapsulated AgOriPVDF BSs with (c) SP-AgOriPVDF electrode and (d) conductive tape electrode. (e) Cyclic fatigue testing: open-circuit output voltage of Ecoflex encapsulated AgOriPVDF BS with SP-AgOriPVDF electrode recorded over $6000 \mathrm{~s}$ (3000 cycles) and magnified data (10 s) after $1000 \mathrm{~s}$ (left insert ) and 5000 s (right insert). All data was recorded under the same conditions (respiration simulator: the compression frequency is $0.5 \mathrm{~Hz}$ and the injected air volume is 250 mL/each).

(a)

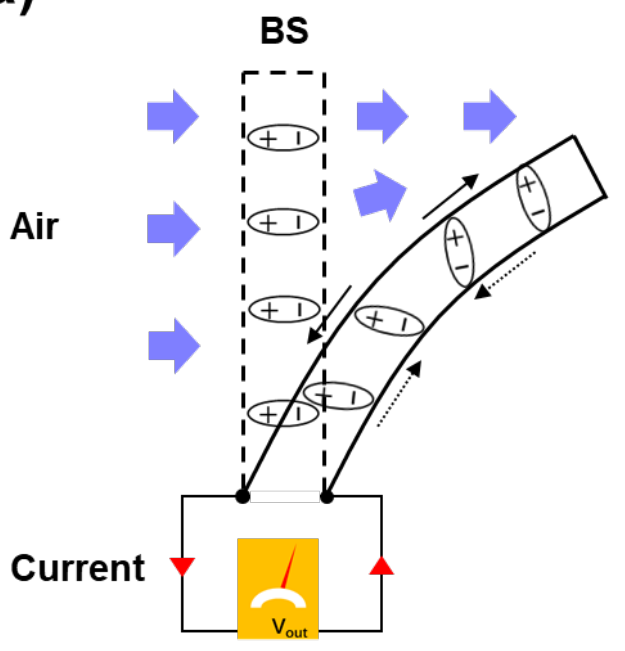

(b)

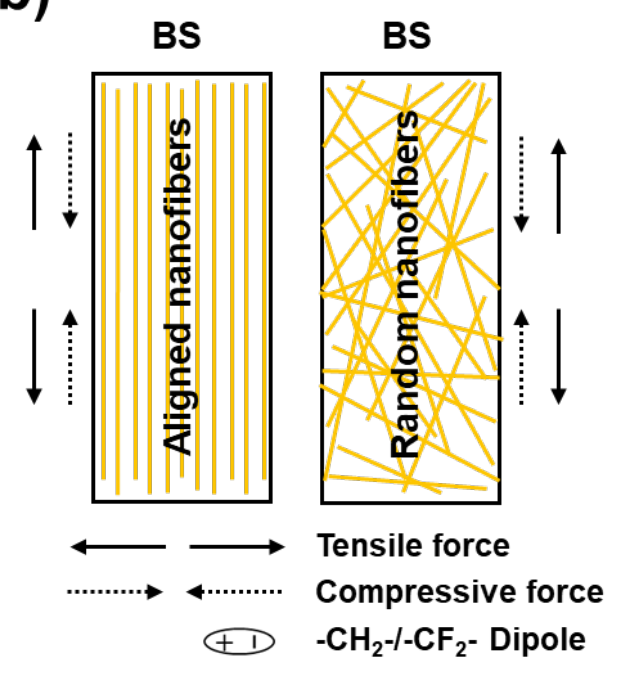



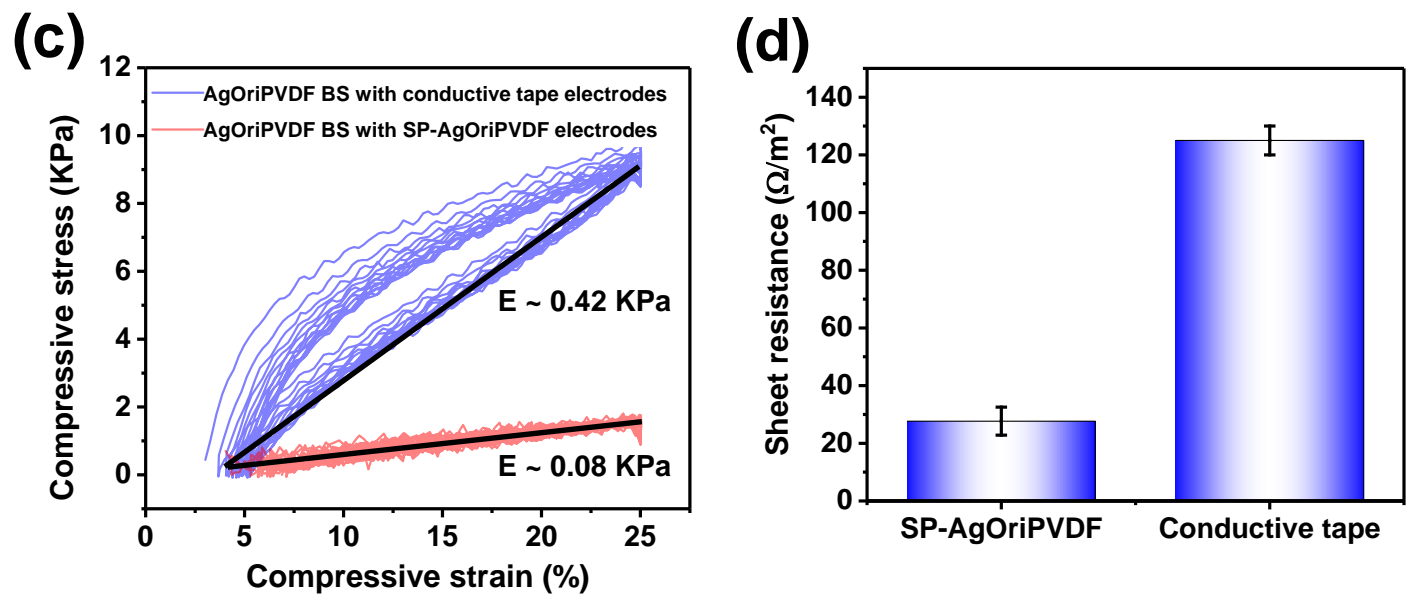

Figure 6. Schematic illustration of (a) a side view of a BS bent by airflow and the resultant dipoles change during bending and (b) comparison of front views of two BS s with aligned nanofibers and random nanofibers and their force diagram. (c) Stressstrain curve (15 cycles) of two encapsulated AgOriPVDF BSs with SP-AgOriPVDF electrode and conductive tape electrode and their compressive elastic modulus (E), and (d) sheet resistances of SP-AgOriPVDF and conductive tape, respectively.

Respiration Measurement. Most piezoelectric materials including piezoelectric PVDF film have pyroelectric property, which means their signal could be influenced by temperature fluctuation, limiting its applications ${ }^{29-31}$. For instance, the piezoelectric PVDF film sensor could be difficult to measure the respiratory signal accurately because of the temperature difference between inhaled and exhaled air. Conversely, the PDVF nanofiber web exhibits relatively low, or almost no, pyroelectricity. As shown Figure 7a, when simultaneously blowing a commercial piezoelectric PVDF film sensor and a piezoelectric PVDF nanofiber web sensor that attached on a white board, the PVDF film sensor shows a clear pyroelectric signal whereas there is no obvious signal generated in the PVDF nanofiber web sensor. This could be attributed to the spongelike nanofiber web structure, which contains the high content of air, hindering that the 
heat conducts into the inside of the nanofiber web.

Therefore, AgOriPVDF BS with SP-AgOriPVDF electrode presents superior piezoelectric bend sensitivity and it has no pyroelectricity at the same time, having a great benefit to measure human respiratory signal. Figure $7 \mathrm{~b}$ demonstrates the respiration measurement setup at open environment. The distance between a subject mouth and sensor is fixed at about $5 \mathrm{~cm}$. The diverse breathing patterns recorded by AgOriPVDF BS is displayed in Figure 7c. Under the slow and light breathing, quite constant $\mathrm{V}_{\mathrm{p}-\mathrm{p}}$ of $\sim 2.5 \mathrm{~V}$ with the peak to peak time interval $\left(\mathrm{T}_{\mathrm{p}-\mathrm{p}}\right)$ of $\sim 1.3 \mathrm{~s}$ is generated, while for rapid and heavy breathing a higher $\mathrm{V}_{\mathrm{p}-\mathrm{p}}(\sim 5 \mathrm{~V})$ with shorter $\mathrm{T}_{\mathrm{p}-\mathrm{p}}(\sim 0.3 \mathrm{~s})$ is measured. It was interesting to find that AgOriPVDF BS could identify coughing from normal breathing. The continuous coughing (three times) detected by AgOriPVDF BS shows inconstant $\mathrm{V}_{\mathrm{p}-\mathrm{p}}$ with a very dense form.

Overall, a simple experimental setup was employed to record human respiratory signal with AgOriPVDF BS. The results showed that AgOriPVDF BS has enough ability to record and identify the breathing patterns such as rate and depth of breathing because of its superior bend sensitivity and excellent flexibility.
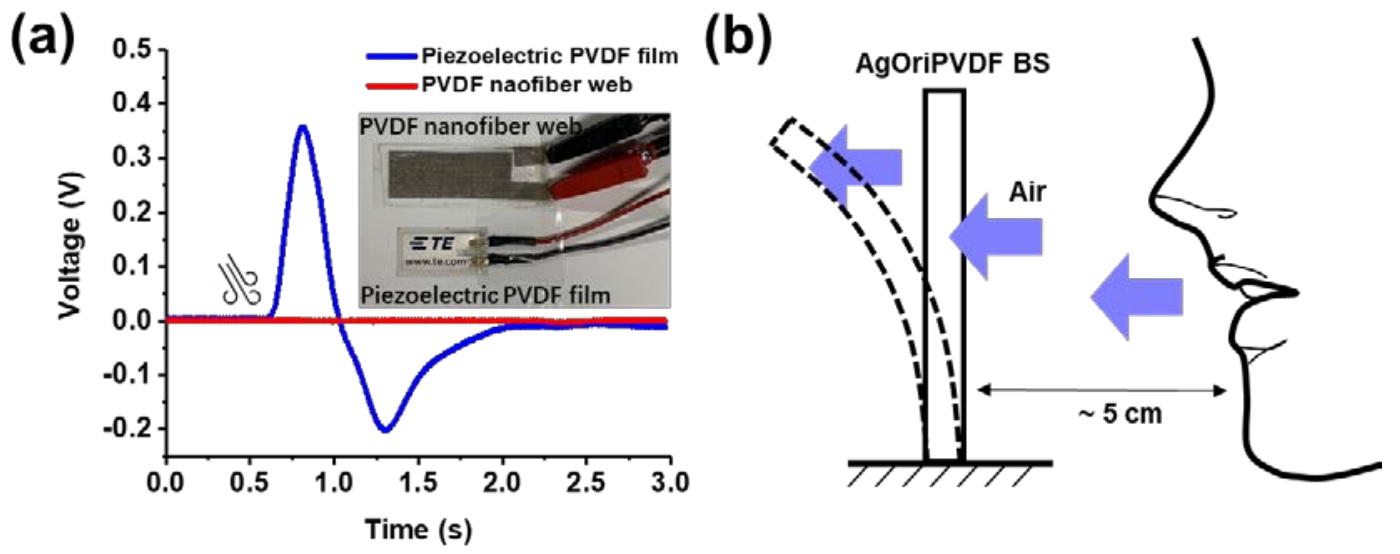


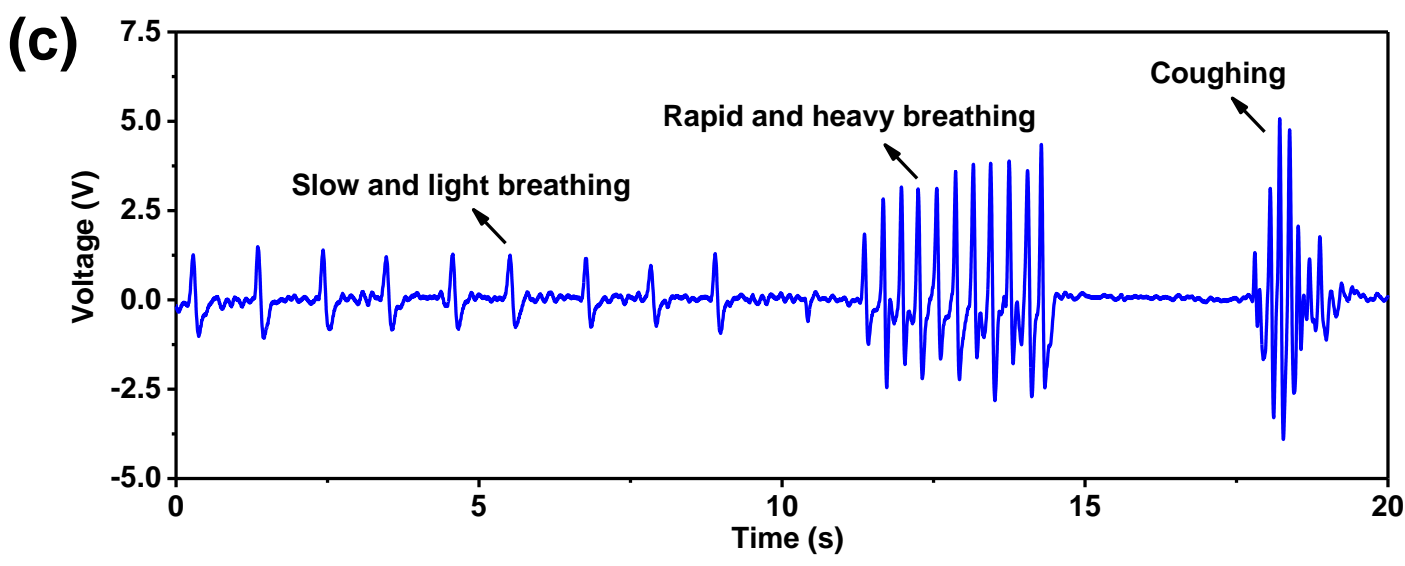

Figure 7. (a) Comparison of pyroelectric response to the expired air between the commercial piezoelectric PVDF film and the electrospun PVDF nanofiber web when slightly blowing the two sensors together attached on a white board with a tape as shown in the inset. (b) Schematic of respiration measurement setup at open environment, in which AgOriPVDF BS is deflected by breathing air. (c) Various breathing patterns detected by AgOriPVDF BS with SP-AgOriPVDF electrode.

\section{CONCLUSIONS}

In summary, we have proved the design strategy to fabricate ultrasensitive BS using PVDF nanofiber web and illustrated its underlying mechanism through analyzing factors i.e. $\beta$-phase content, nanofiber orientation and flexibility of electrode. The unique electrospinning ( $\mathrm{RSC}=2000 \mathrm{rpm}$ and $\mathrm{TCD}=4 \mathrm{~cm}$ ) with silver nanoparticles interfacing results in the Ag-doped oriented PVDF nanofiber web with high $\beta$-phase content $\left(\mathrm{C}_{\beta} \sim 44.5 \%\right)$ i.e. AgOriPVDF. Consequently, the piezoelectric bending response towards airflow of AgOriPVDF $\left(\mathrm{V}_{\mathrm{p}-\mathrm{p}} \approx 0.2 \mathrm{~V}\right)$ increases 10 times in comparison to that of the random aligned NeatPVDF $\left(\mathrm{V}_{\mathrm{p}-\mathrm{p}} \approx 0.02 \mathrm{~V}\right)$. Moreover, due to the highly flexible electrode made of AgOriPVDF via electroless silver plating process i.e. SP-AgOriPVDF, the Ecoflex encapsulated AgOriPVDF BS with SP-AgOriPVDF 
electrode $\left(\mathrm{V}_{\mathrm{p}-\mathrm{p}} \approx 4.6 \mathrm{~V}\right)$ possesses 3 times higher piezoelectric response than that with conductive tape electrode $\left(\mathrm{V}_{\mathrm{p}-\mathrm{p}} \approx 1.4 \mathrm{~V}\right)$. As a whole, the piezoelectric bending response of encapsulated AgOriPVDF BS with SP-AgOriPVDF electrode $\left(\mathrm{V}_{\mathrm{p}-\mathrm{p}} \approx 4.6 \mathrm{~V}\right)$ elevates more than 200 times compared to that of the unpackaged NeatPVDF BS with conductive tape electrode $\left(\mathrm{V}_{\mathrm{p}-\mathrm{p}} \approx 0.02 \mathrm{~V}\right)$. Finally, we have demonstrated that the AgOriPVDF BS has sufficient capacity to detect and identify the respiratory signal. Thanks to the superior bend sensitivity, the proposed AgOriPVDF BS will have an immense possibility for wearable applications, e.g. smart respiratory protective equipment.

\section{ASSOCIATED CONTENT}

Supporting Information

The Supporting Information is available free of charge on the ACS Publications website at DOI:

Equation for crystallinity calculation, cyclic fatigue bending testing for SP-AgOriPVDF, photograph of the experiment setup to measure the output voltage with the respiration simulator and Instron, $\mathrm{C}_{\mathrm{t}}, \mathrm{C}_{\beta}$ and Vp-p of Ag doped randomly oriented PVDF nanofiber web, comparison of piezoelectric pressure response of two encapsulated AgOriPVDF BSs with SP-AgOriPVDF electrode and conductive tape electrode.

\section{AUTHOR INFORMATION}

Corresponding Author

*E-mail: henry.yili@manchester.ac.uk

ORICD 
Yi Li: 0000-0002-2092-4505

Lu Jin: 0000-0002-4857-2206

Zekun Liu: 0000-0002-8482-7456

Jiashen Li: 0000-0001-7333-5280

Notes

The authors declare no competing financial interest.

\section{ACKNOWLEDGEMENTS}

We would like to acknowledge the support of British Cotton Growers’ Association

Work People’s Collection Fund with the project “Protective Efficiency of Respiratory

Protective Equipment against Byssinosis for Cotton Workers” and University of Manchester through project AA14512 (UMRI project "Graphene-Smart Textiles EHealthcare Network”). L. J. and Z. L. were funded by the China Scholarship Council (CSC).

\section{REFERENCES}

(1) Maity, K.; Mandal, D. All-Organic High-Performance Piezoelectric Nanogenerator with Multilayer Assembled Electrospun Nanofiber Mats for Self-Powered Multifunctional Sensors. ACS Appl. Mater. Interfaces 2018, 10 (21), 18257-18269. https://doi.org/10.1021/acsami.8b01862.

(2) Sengupta, D.; Kottapalli, A. G. P.; Chen, S. H.; Miao, J. M.; Kwok, C. Y.; Triantafyllou, M. S.; Warkiani, M. E.; Asadnia, M. Characterization of Single Polyvinylidene Fluoride (PVDF) Nanofiber for Flow Sensing Applications. AIP Adv. 2017, 7 (10), 105205. https://doi.org/10.1063/1.4994968. 
(3) Chorsi, M. T.; Curry, E. J.; Chorsi, H. T.; Das, R.; Baroody, J.; Purohit, P. K.; Ilies, H.; Nguyen, T. D. Piezoelectric Biomaterials for Sensors and Actuators. Adv. Mater. 2019, 31 (1), 1-15. https://doi.org/10.1002/adma.201802084.

(4) Wang, X.; Sun, F.; Yin, G.; Wang, Y.; Liu, B.; Dong, M. Tactile-Sensing Based on Flexible PVDF Nanofibers via Electrospinning: A Review. Sensors (Switzerland) 2018, 18 (2), 330. https://doi.org/10.3390/s18020330.

(5) Garain, S.; Jana, S.; Sinha, T. K.; Mandal, D. Design of in Situ Poled Ce3+Doped Electrospun PVDF/Graphene Composite Nanofibers for Fabrication of Nanopressure Sensor and Ultrasensitive Acoustic Nanogenerator. ACS Appl. Mater. Interfaces 2016, 8 (7), 4532-4540. https://doi.org/10.1021/acsami.5b11356.

(6) Fortunato, M.; Cavallini, D.; De Bellis, G.; Marra, F.; Tamburrano, A.; Sarto, F.; Sarto, M. S. Phase Inversion in PVDF Films with Enhanced Piezoresponse through Spin-Coating and Quenching. Polymers (Basel). 2019, 11 (7), 1-15. https://doi.org/10.3390/polym11071096.

(7) Wang, Y. R.; Zheng, J. M.; Ren, G. Y.; Zhang, P. H.; Xu, C. A Flexible Piezoelectric Force Sensor Based on PVDF Fabrics. Smart Mater. Struct. 2011, 20 (4), 045009. https://doi.org/10.1088/0964-1726/20/4/045009.

(8) Lee, S. J.; Prabu, A. A.; Jin, K. Piezoelectric Properties of Electrospun Poly (LLactic Acid ) Nanofiber Web. Mater. Lett. 2015, 148, 58-62. https://doi.org/10.1016/j.matlet.2015.02.038.

(9) Ding, B.; Wang, M.; Wang, X.; Yu, J.; Sun, G. Electrospun Nanomaterials for 
Ultrasensitive Sensors. Mater. Today 2010, 13 (11), 16-27.

https://doi.org/10.1016/S1369-7021(10)70200-5.

(10) Nasir, M.; Kotaki, M. Fabrication of Aligned Piezoelectric Nanofiber By

Electrospinning. Int. J. Nanosci. 2009, 08 (03), 231-235.

https://doi.org/10.1142/S0219581X09006092.

(11) Shao, H.; Fang, J.; Wang, H.; Lin, T. Effect of Electrospinning Parameters and Polymer Concentrations on Mechanical-to-Electrical Energy Conversion of Randomly-Oriented Electrospun Poly(Vinylidene Fluoride) Nanofiber Mats. RSC Adv. 2015, 5 (19), 14345-14350. https://doi.org/10.1039/c4ra16360e.

(12) Gee, S.; Johnson, B.; Smith, A. L. Optimizing Electrospinning Parameters for Piezoelectric PVDF Nanofiber Membranes. J. Memb. Sci. 2018, 563

(February), 804-812. https://doi.org/10.1016/j.memsci.2018.06.050.

(13) Jiyong, H.; Yinda, Z.; Hele, Z.; Yuanyuan, G.; Xudong, Y. Mixed Effect of Main Electrospinning Parameters on the $\beta$-Phase Crystallinity of Electrospun PVDF Nanofibers. Smart Mater. Struct. 2017, 26 (8), 085019.

https://doi.org/10.1088/1361-665X/aa7245.

(14) Wan, C.; Bowen, C. R. Multiscale-Structuring of Polyvinylidene Fluoride for Energy Harvesting: The Impact of Molecular-, Micro- and Macro-Structure. $J$. Mater. Chem. A 2017, 5 (7), 3091-3128. https://doi.org/10.1039/c6ta09590a.

(15) Xin, Y.; Qi, X.; Tian, H.; Guo, C.; Li, X.; Lin, J.; Wang, C. Full-Fiber Piezoelectric Sensor by Straight PVDF/Nanoclay Nanofibers. Mater. Lett. 2016, 164, 136-139. https://doi.org/10.1016/j.matlet.2015.09.117. 
(16) Abbasipour, M.; Khajavi, R.; Yousefi, A. A.; Yazdanshenas, M. E.; Razaghian, F. The Piezoelectric Response of Electrospun PVDF Nanofibers with Graphene Oxide, Graphene, and Halloysite Nanofillers: A Comparative Study. J. Mater. Sci. Mater. Electron. 2017, 28 (21), 15942-15952. https://doi.org/10.1007/s10854-017-7491-4.

(17) Yang, D.; Lu, B.; Zhao, Y.; Jiang, X. Fabrication of Aligned Fibrous Arrays by Magnetic Electrospinning. Adv. Mater. 2007, 19 (21), 3702-3706. https://doi.org/10.1002/adma.200700171.

(18) Yu, L.; Shao, Z.; Xu, L.; Wang, M. High Throughput Preparation of Aligned Nanofibers Using an Improved Bubble-Electrospinning. Polymers (Basel). 2017, 9 (12), 1-9. https://doi.org/10.3390/polym9120658.

(19) Kim, H. S.; Park, I. K. Enhanced Output Power from Triboelectric Nanogenerators Based on Electrospun Eu-Doped Polyvinylidene Fluoride Nanofibers. J. Phys. Chem. Solids 2018, 117, 188-193. https://doi.org/10.1016/j.jpcs.2018.02.045.

(20) Parangusan, H.; Ponnamma, D.; Al-Maadeed, M. A. A. Stretchable Electrospun PVDF-HFP/Co-ZnO Nanofibers as Piezoelectric Nanogenerators. Sci. Rep. 2018, 8 (754), 1-11. https://doi.org/10.1038/s41598-017-19082-3.

(21) Mandal, D.; Yoon, S.; Kim, K. J. Origin of Piezoelectricity in an Electrospun Poly(Vinylidene Fluoride-Trifluoroethylene) Nanofiber Web-Based Nanogenerator and Nano-Pressure Sensor. Macromol. Rapid Commun. 2011, 32 (11), 831-837. https://doi.org/10.1002/marc.201100040. 
(22) Zeng, W.; Tao, X.-M.; Chen, S.; Shang, S.; Chan, H. L. W.; Choy, S. H. Highly Durable All-Fiber Nanogenerator for Mechanical Energy Harvesting. Energy Environ. Sci. 2013, 6 (9), 2631. https://doi.org/10.1039/c3ee41063c.

(23) Ghosh, S. K.; Mandal, D. Synergistically Enhanced Piezoelectric Output in Highly Aligned 1D Polymer Nanofibers Integrated All-Fiber Nanogenerator for Wearable Nano-Tactile Sensor. Nano Energy 2018, 53, 245-257. https://doi.org/10.1016/j.nanoen.2018.08.036.

(24) Cai, X.; Lei, T.; Sun, D.; Lin, L. A Critical Analysis of the $\alpha, \beta$ and $\gamma$ Phases in Poly(Vinylidene Fluoride) Using FTIR. RSC Adv. 2017, 7 (25), 15382-15389. https://doi.org/10.1039/c7ra01267e.

(25) Mandal, D.; Henkel, K.; Schmeißer, D. Improved Performance of a Polymer Nanogenerator Based on Silver Nanoparticles Doped Electrospun P(VDFHFP) Nanofibers. Phys. Chem. Chem. Phys. 2014, 16 (22), 10403. https://doi.org/10.1039/c3cp55238a.

(26) Martins, P.; Lopes, A. C.; Lanceros-Mendez, S. Electroactive Phases of Poly(Vinylidene Fluoride): Determination, Processing and Applications. Prog. Polym. Sci. 2014, 39 (4), 683-706. https://doi.org/10.1016/j.progpolymsci.2013.07.006.

(27) Selvan, R. T.; Ahn, Y. J.; Kim, K. J.; Kim, H. Uniaxially Drawn Polylactic Acid Film Based Physiological Sensor for Monitoring Sleeping Parameters. Fibers Polym. 2017, 18 (10), 1898-1905. https://doi.org/10.1007/s12221-0177563-3. 
(28) Logeeswaran, V. J.; Katzenmeyer, A.; Islam, M. S.; Kobayashi, N. P.; Wu, W.; Chaturvedi, P.; Fang, N.; Wang, S. Y.; Williams, R. S. Electrical Resistivity \& Thermal Stability of Smooth Silver Thin Film for Nanoscale Optoelectronic Devices. 2008 8th IEEE Conf. Nanotechnology, IEEE-NANO 2008, 92-94. https://doi.org/10.1109/NANO.2008.34.

(29) Ando, M.; Kawamura, H.; Kitada, H.; Sekimoto, Y.; Inoue, T.; Tajitsu, Y. Pressure-Sensitive Touch Panel Based on Piezoelectric Poly(L-Lactic Acid) Film. Jpn. J. Appl. Phys. 2013, 52, 09KD171-4. https://doi.org/10.7567/JJAP.52.09KD17.

(30) Wu, Y.; Du, X.; Gao, R.; Li, J.; Li, W.; Yu, H.; Jiang, Z.; Wang, Z.; Tai, H. Self-Polarization of PVDF Film Triggered by Hydrophilic Treatment for Pyroelectric Sensor with Ultra-Low Piezoelectric Noise. Nanoscale Res. Lett. 2019, 14, 1-9. https://doi.org/10.1186/s11671-019-2906-1.

(31) Bae, J.-H.; Chang, S.-H. PVDF-Based Ferroelectric Polymers and Dielectric Elastomers for Sensor and Actuator Applications: A Review. Funct. Compos. Struct. 2019, 1 (1), 012003. https://doi.org/10.1088/2631-6331/ab0f48.

\section{Table Of Contents}



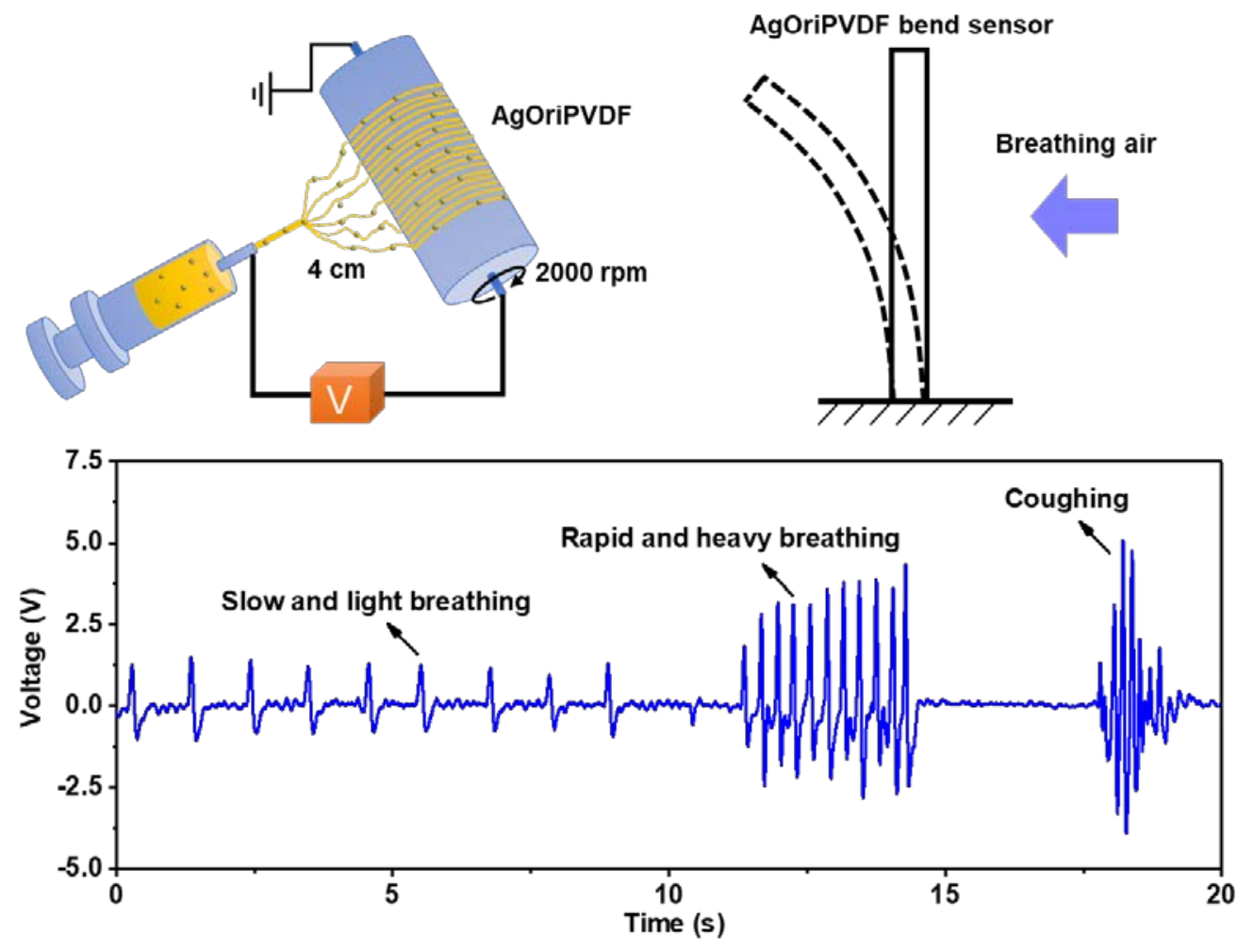